\title{
Role of autophagy in regulation of glioma stem cells population during therapeutic stress
}

\author{
Abbas $\mathrm{S}^{1 \#}$, Singh $\mathrm{SK}^{2 \#}$, Saxena $\mathrm{AK}^{2}$, Tiwari $\mathrm{S}^{1}$, Sharma $\mathrm{LK}^{1}$, Tiwari $\mathrm{M}^{2}$
}

\begin{abstract}
Glioblastoma is highly recurrent and aggressive tumor with poor prognosis where existence of glioma stem cell (GSCs) population is well established. The GSCs display stem cell properties such as self-renewable, proliferation and therapeutic resistance which contribute to its role in tumor progression, metastasis and recurrence. Cancer stem cells (CSCs) can also be induced from non-stem cancer cells in response to radio/chemotherapy that further contribute to cancer relapse post therapy. Role of autophagy has been implicated in the existence of CSCs in different cancers; however, its role in GSCs is still unclear. Moreover, since autophagy is induced in response to various chemotherapeutic agents, it becomes imperative to understand the role of autophagy in therapy-induced pool of CSCs. Here, we investigated the role of autophagy in the maintenance of GSCs and temozolomide (TMZ)-induced therapeutic response. Glioblastoma cell lines (U87MG, LN229) were cultured as monolayer as well as GSC enriched tumorspheres and sub-spheroid population. Our results demonstrated that the tumorspheres maintained higher level of autophagy than the monolayer cells and inhibition of autophagy significantly reduced the percentage of GSCs and their self-renewal capacity. Further, TMZ at clinically relevant concentration resulted in an induction of survival autophagy in glioblastoma cells. We also observed that TMZ treatment significantly increased the expression of GSC markers, suggesting an increased pool of GSCs. Importantly, inhibition of autophagy prevented this TMZ-induced increased GSC population, suggesting a critical role for autophagy in therapy-induced generation of GSC pool. Overall, our findings revealed; i) higher levels of autophagy in GSCs; ii) TMZ induces protective autophagy and up-regulates pool of GSCs; and iii) inhibition of autophagy prevents TMZ-induced GSCs pool suggesting its role regulating GSC population in response to chemotherapy. Our study signifies a positive contribution of autophagy in survival of GSCs which implicates the use of autophagy inhibitors in a combinational approach to target TMZ-induced GSCs for developing effective therapeutic strategies. Further efforts are required to study the role of autophagy in therapy- induced GSC pool in other cancer types for its broad therapeutic implication.
\end{abstract}

Key Words: Glioblastoma; Autophagy: Glioma stem cells; Temozolomide; Therapy-induced glioma stem cells

\section{Introduction}

High grade gliomas (HGGs) are extremely malignant brain tumors that are associated with poor prognosis and severely affect quality of life and cognitive function of its patients ${ }^{[1]}$. Despite availability of multimodal therapeutic regime, recurrence of such tumor is unavoidable due to its invasive behavior and resistance to therapeutic interventions. Till date, temozolomide (TMZ) in combination with localized radiation therapy is the best known therapeutic option for treating $\mathrm{HGG}^{[2]}$. TMZ, a known alkylating agent shows antitumor effect by formation of methyl adducts at $\mathrm{N}^{7}$ and $\mathrm{O}^{6}$ positions of guanine and the $\mathrm{N}^{3}$ of adenine $e^{[3]}$. However, this adduct can be removed by a DNA repair enzyme $\mathrm{O}^{6}$ methylguanine-DNA methyltransferase enzyme (MGMT) and causes resistance in glioblastoma ${ }^{[4]}$. TMZ can extend the life of the patients up to 2 years; still, long term survival is rare due to resistance and recurrence ${ }^{[2,3]}$. Several researchers have attributed such resistance and recurrence of tumors to the existence of cancer stem cells $(\mathrm{CSCs})^{[5,6]}$. It has been identified that CSCs exist in solid tumors including glioma and similar to stem cells possess ability of self-renewal and differentiation into heterogeneous lineages of cancer cells. These CSC constitute a small population within tumor and are responsible for tumor progression, resistance to therapy, recurrence and aggressive behavior of the tumor. Thus, targeting CSC becomes imperative for effective treatment of cancer leading to progression free survival ${ }^{[5,6]}$.

Autophagy is a catabolic process where cellular metabolites and components are recycled through lysosomal degradation and contribute to cellular survival during cellular stress under physiological and pathological conditions. It is also induced by cancer therapy which may contribute to therapeutic resistance and long periods of dormancy followed by metastatic disease. Role of autophagy has also been implicated in the functioning of CSC in a variety of tumors ${ }^{[7]}$, however, its contribution in modulating CSC population during therapeutic stress remains elusive. Due to its clinical implications, the mechanisms by which autophagy contributes to stemness and maintenance of CSC during therapeutic stress is of great research interest. Previous studies demonstrated role of autophagy inhibition in enhancement of chemotherapeutic potential of TMZ in glioblastoma cell lines and animal models; such combinations are also under clinical trials ${ }^{[8-10]}$. Interestingly, although, TMZ at clinically achievable plasma and cerebrospinal fluid concentrations can prevent proliferation of tumor population, but also promotes the growth of glioma stem cell (GSC) population $^{[11]}$. Such GSC population might be responsible for tumor recurrence and chemoresistance. Therefore, in the present study we 
hypothesize that autophagy plays a crucial role in maintenance of GSC and inhibition of autophagy may be considered as a promising strategy for sensitizing CSCs to anticancer therapy such as TMZ.

Our study identifies that GSCs maintains higher level of autophagy as compared to parental monolayer cells. Autophagy is also induced in response to clinically relevant TMZ concentrations and provides survival advantage. Importantly, autophagy plays an essential role in TMZ-induced generation of GSC population. Thus, autophagy inhibition could be an alternative approaches to sensitize glioblastoma cells towards chemotherapy and to prevent the generation of GSC pool contributing in tumor aggressiveness and recurrence.

\section{Material and Methods}

\section{Cell culture}

Glioblastoma cell lines U87MG and LN229 were procured from National repository at National Centre for Cell Science, Pune, India and cultured in Minimal Essential Medium (MEM) or Dulbecco's Modified Eagle Medium (DMEM) respectively (Gibco) with nonessential amino acids, containing glutamine $(4 \mathrm{mM})$, and penicillinstreptomycin $(50 \mathrm{IU} / \mathrm{ml})$ with FBS $(10 \%), 1.5 \mathrm{~g} / 1$ sodium bicarbonate, and $4.5 \mathrm{~g} / 1$ glucose at $37^{\circ} \mathrm{C}, 5 \% \mathrm{CO}_{2}$ and $95 \%$ relative humidity.

\section{In-vitro propagation of tumor-initiating spheroids}

Tumor-initiating spheroids (tumorspheres) were generated through 3 dimensional (3-D) culture of human glioblastoma cells U87MG and LN229 cells under low attachment conditions. Different concentrations of agarose (0.1-1.5\%) were pre-coated in 96/24/12 well plates and varying numbers of cells were seeded to optimize low attachment conditions for spheroids formation, following previously published protocol ${ }^{[12]}$. Cells were incubated in a humidified atmosphere with $5 \% \mathrm{CO}_{2}$ at $37^{\circ} \mathrm{C}$ for 7 days. These spheroids ranging from $100-250 \mu \mathrm{m}$ in size were collected by gentle centrifugation and further propagated to obtain sub-spheroids. The formation of tumor spheres was observed under phase-contrast inverted microscope every day (Olympus IX73) with color CCD camera. Images from random fields were captured and number and size of spheres formed in each well was determined after a week and analyzed using the Olympus cellSens software. The number and size of tumorspheres were determined from at least three independent experiments.

\section{Side population $(S P)$ analysis}

For SP assay, previously published protocol was followed with minor modifications $^{[13]}$. The cells were treated with autophagy inhibitors, chloroquine (CQ) and 3-methyl adenine (3-MA) or treated with carrier control for 72 hrs. The cells were harvested and $\sim 10^{6}$ cells $/ \mathrm{ml}$ were resuspended in Hank's balanced salt solution containing 2\% FBS and $10 \mathrm{mM}$ HEPES. The cells were incubated in the presence or absence of Verapamil $(100 \mu \mathrm{M})$ for $60 \mathrm{~min}$ at $37^{\circ} \mathrm{C}$ with intermittent shaking followed by Hoechst-33342 $(10 \mu \mathrm{g} / \mathrm{ml})$ staining for $15 \mathrm{~min}$ and fluorescence was analyzed at $450 / 675 \mathrm{~nm}$ (Hoechst blue/red) detection wavelength. Dead cells were excluded using PI staining (10 $\mu \mathrm{g} / \mathrm{ml}$, incubated for $10 \mathrm{~min}$ ). The cells identified inside the lowHoechst blue and red area without Verapamil as the side population. Data was analyzed using Attune NxT Flow Cytometer Software. Experiment was performed in triplicates.

\section{Cell proliferation and viability}

The effect of various treatments (TMZ, autophagy inhibitors 3-MA and CQ or combinations) on cell proliferation/inhibition was studied in the parental adherent monolayer and tumorspheres by using 3-[4,5-dimethylthiazol-2-yl]-2,5 diphenyl tetrazolium bromide (MTT) assay which is based on the conversión of MTT into formazan crystals in the living cells in mitochondria and thus indicate live and proliferating cells. Tumorspheres were developed by plating 1500 cells/well in 96 well plates, in triplicates in low attachment plates and treatment was initiated after $24 \mathrm{hrs}$ of plating. The spheres were treated for 7 days and half the media was changed on the third day of treatment. Single cell suspension was prepared and the cells were transferred in 96 well plates for MTT/PI assay. For adherent cells $2 \times 10^{3}$ cells per well were seeded in 96-well plates in $200 \mu$ culture medium (three wells per group). Following $24 \mathrm{hrs}$, the cells were treated with various concentrations of drugs for indicated time. MTT reagent $(20 \mu \mathrm{l}$ from $5 \mathrm{mg} / \mathrm{ml})$ solution was added in the media and incubated for another $4 \mathrm{hrs}$ at $37^{\circ} \mathrm{C}$. The cells were lysed in buffer solution containing $10 \%$ sodium dodecyl sulfate (SDS) in $0.01 \mathrm{M} \mathrm{HCl}$ buffer solution. The absorbance was read at $570 \mathrm{~nm}$. Cell viability (\%) was determined using the following formula: $\%$ cell viability $=\left(\mathrm{A}_{570}\right.$ of treated cells $/ \mathrm{A}_{570}$ of control cells $) \times 100$. Since MTT is based on mitochondrial activity which may also be reduced due to inhibition of cellular proliferation, PI staining $(10 \mu \mathrm{g} / \mathrm{ml}$ for $30 \mathrm{~min})$ was also performed in parallel to analyze dead cells and normalized with total number of nuclei stained with Hoechst-33258 $(10 \mu \mathrm{g} / \mathrm{ml})$ staining to calculate percentage cell death.

Measurement of intracellular reactive oxygen species (ROS) and mitochondrial membrane potential (MMP) and Acidic vacuolar organelles (AVO)

After treatments, single cell suspensions were prepared from adherent cells or tumorspheres (containing GSCs) and transferred to a new plate for further assay. Staining of cells/spheroids with various dyes that included 2',7'-Dichlorodihydrofluorescein diacetate $\left(\mathrm{H}_{2}\right.$ DCFDA $)(10 \mu \mathrm{M})$ or Mitoview $^{\mathrm{TM}} 633 \quad(5 \mu \mathrm{M})$ or Acridine Orange (AO) $10 \mu \mathrm{g} / \mathrm{ml}$ or PI $(10 \mu \mathrm{g} / \mathrm{ml})$ and Hoechst $33258(10 \mu \mathrm{g} / \mathrm{ml})$ was performed for $15-30 \mathrm{~min}$ at $37^{\circ} \mathrm{C}$ in dark with mild shaking to prevent clumping. The cells were pelleted and washed to remove any unbound dye. The cells were re-suspended and immediately analyzed using a fluorescent plate reader at appropriate wavelengths (DCFDA: 492-95/512-527 nm, Mitoview $^{\operatorname{Tm}} 633$ : $622 / 648 \mathrm{~nm}$, AO: 460/650 nm, PI: 493/636 nm, Hoechst: $350 / 461 \mathrm{~nm}$ ).

\section{Immuno-fluorescence microscopy}

For immunofluorescence analysis, GSCs, GSC-spheres and or parental cells were seeded on glass coverslips, allowed to adhere and were fixed with $4 \%$ paraformaldehyde in PBS for $30 \mathrm{~min}$ at room temperature (RT), permeabilized with $0.3 \%$ Triton X-100 for 5 min and blocked in $4 \%$ bovine serum albumin or serum in PBS. Cells were incubated over night with primary antibody $(1: 200)$ at $4^{\circ} \mathrm{C}$ with gentle shaking (list with catalogue number of antibodies provided in supplementary material). The coverslips were washed three times with PBS followed by incubation with fluorescent-labeled secondary antibody for $1 \mathrm{hr}$ at RT. Coverslips were washed and stained with Hoechst-33258 for 5 min, mounted on a slide using antifade reagent VectaShield (Vector Labs) and sealed with nail polish to prevent drying and movement under microscope. The slides were kept at $4{ }^{\circ} \mathrm{C}$, protected from light and were analysis within a week. Slides were examined using Olympus Fluoview FV-10i confocal laserscanning microscope or Olympus IX73) with color CCD camera at 40X magnification. The images were then processed with ImageJ (http://rsb.info.nih.gov/ij/) software.

\section{Immunoblotting}

Western blotting was performed following established protocol in our laboratory ${ }^{[14]}$. Control and treated monolayer cultures or GSCs tumorspheres were suspended in RIPA lysis buffer, containing 20 $\mathrm{mM}$ Tris- $\mathrm{HCl}(\mathrm{pH} 7.5), 150 \mathrm{mM} \mathrm{NaCl}, 1 \mathrm{mM}$ Na2EDTA, $1 \mathrm{mM}$ EGTA $1 \%$ NP-40, $1 \%$ sodium deoxycholate, $2.5 \mathrm{mM}$ sodium pyrophosphate, $1 \mathrm{mM} \beta$-glycerophosphate, $1 \mathrm{mM} \mathrm{Na}_{3} \mathrm{VO}_{4}, 1 \mu \mathrm{g} / \mathrm{ml}$ 
Leupeptin, 5mM phenylmethylsulfonyl fluoride incubated on ice for $30 \mathrm{~min}$ followed by lysis using ultrasonicator (frequency of $20 \mathrm{kHz}$ ), one time for 2-3 minutes on ice. The lysate was then centrifuged at $14000 \mathrm{rpm}$ for 30 minutes at $4{ }^{\circ} \mathrm{C}$. After addition of protease inhibitors, the lysates were stored at $-20{ }^{\circ} \mathrm{C}$ until further analysis. Protein estimation was performed using the BCA kit (Pierce). Equal amounts of protein from the total cell lysates (40- $50 \mu \mathrm{g} / \mathrm{lane})$ were separated by 10 to $12 \%$ sodium-dodecyl sulphate polyacrilamide gel electrophoresis (SDS-PAGE) analysis. The proteins were transferred on polyvinilidenedifluoride (PVDF) membranes (Bio-Rad, Hercules, California, USA) and after blocking with BSA or Non fat dry milk, were probed with various primary antibodies (for details supplementary file) after following manufactures protocol. After washing three times with tris-buffered saline with Tween-20 (TBST) the blots were probed with HRP labeled secondary antibody (1:2000) for $2 \mathrm{hrs}$. After washing, the bands were visualized by chemiluminescence reaction, using the ECL Western detection system (Millipore, Darmstadt, Germania), for stem cell markers Amersham ECL Select Western Blotting Detection Reagent (RPN2235) was used. Densitometric analysis was performed by Image J software (NIH-USA).

\section{Statistical analysis}

Data is presented as mean \pm standard deviation (SD). Data were analyzed using Student's $t$ test or one-way ANOVA with Bonferroni posttests, $* P<0.05, * * P<0.01$ indicate a statistically significant difference (Graph Pad Prism 5 Software, San Diego, CA, USA).

\section{Results}

Tumorspheres as a model for GSC

To determine the level of autophagy and its role in GSCs, a wellestablished in-vitro svstem in which enriched GSCs can be propagated as floating spherical colonies as tumorspheres was employed $^{[15]}$. U87MG and LN229, cell lines of human origin derived from glioblastoma patients carrying normal or mutated form of $\mathrm{p} 53$, were employed in the study. These cell lines are commonly used to study drug cytotoxicity due to their intact apoptotic and autophagic machinery and also contain GSC population. The cell lines were maintained as monolayer under standard conditions (Figure 1A). For enrichment of GSC population from the parental cell lines (U87MG, LN229), the cells were cultured as spheroid in CSC media in the absence of serum, supplemented with growth factors FGF-2, EGF and B27 under non-adherent culture conditions using agarose coated plates $^{[12]}$. Among different agarose concentrations, 0.9\% agarose bedding was found the most suitable for generation of GSC enriched spheroids, as can be seen with an increased protein expression of GSC marker CD133 in spheroid cultures than in the monolayer culture (Figure 1B). Accordingly, there was loss of differentiation marker beta-3-tubulin (highest loss at $0.9 \%$ agarose bedding), suggesting enrichment of CSC in spheroids compared with the monolayer (Figure 1B). Thus, $0.9 \%$ agarose coated plates were used for spheroid generation for further studies. Further, the number of cells for optimum spheroid formation was also determined (in a 96 well plate ranged from 500-2000 cells/well in $200 \mu \mathrm{L}$ media (data not shown)). The average number of tumorspheres formed varied based on the number plated with a size between 100-250 $\mu \mathrm{m}$ when plated at a density of 1500 cell/ well in 96 well plate after 7 days (Figure 1C-D). These tumorspheres could be propagated in to subtumorspheres and retain the self-renewal and proliferation capability, a property of GSC (Figure 1D). Further to confirming the property of GSC in these tumorspheres, immunocytochemistry was also performed using bonafide GSC markers SSEA1/CD15 a cell surface marker and pluripotency markers NANOG and SOX2 in GSCs (Figure 1E-F). Results demonstrated that the tumorspheres expressed higher stem makers, such as CD15, NANOG as well as SOX2, suggesting enrichment of cancer stem-like cells in these spheroids.
A
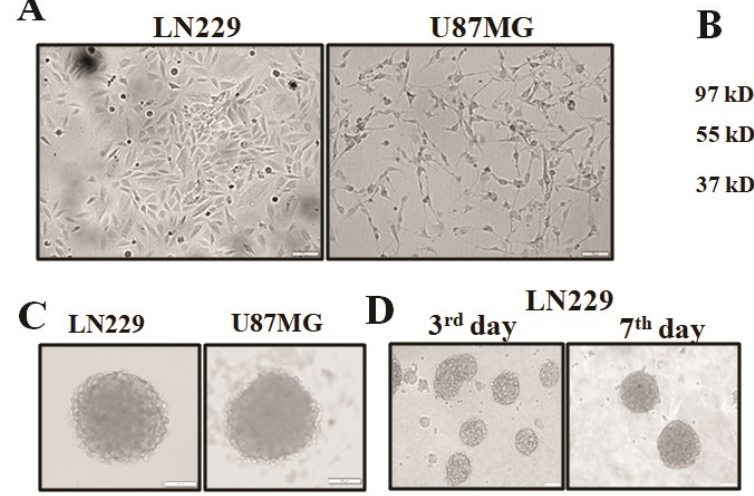

$\mathbf{E}$

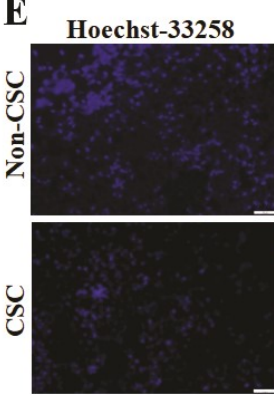

CD15/SSEA1
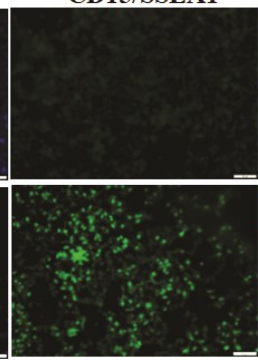
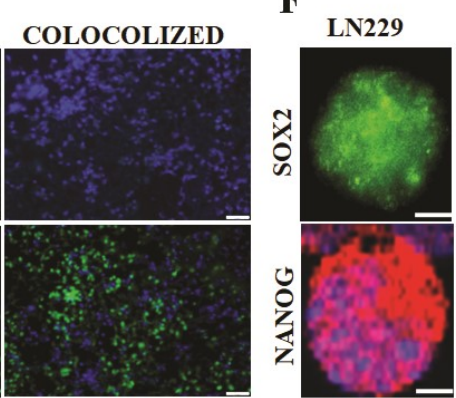

B
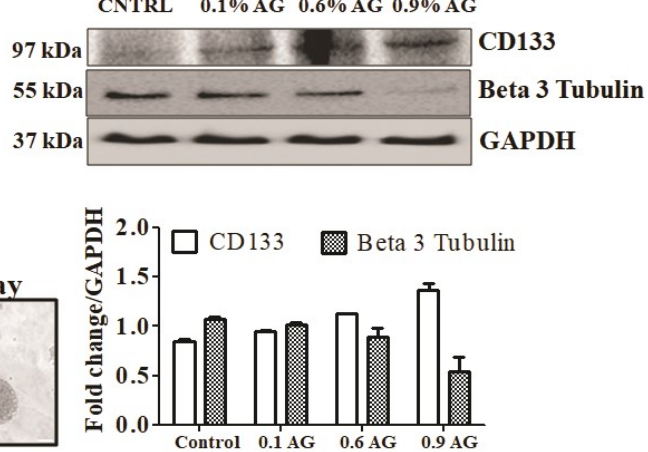

U87MG

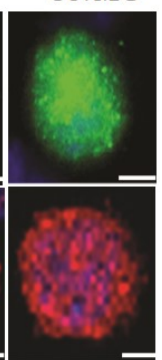

Figure. 1 Tumorspheres generation from parental cells.

A Epithelial-like morphology of the parental LN229 and U87MG adherent monolayer, cultured in DMEM and MEM medium respectively with $10 \%$ FBS. B Tumorsphere were generated from LN229 cells (1x1 $0^{4}$ cells $/ \mathrm{mL}$ ) in CSC media low attachment agarose (0.01-0.9\%) coated wells. Western blotting for tumorspheres shows enrichment of GSC markers CD133 and loss of differentiation marker beta-3-tubulin, GAPDH as loading control, lane 1-CNTRL are the parental LN229, and lane 2-4 represents range of agarose concentration for growing tumorspheres. C Tumorspheres from 1500 cells /well in 96 well plate (LN229 and U87MG cells) were grown in non-adherent suspension CSC growth, presenting smooth-edged spherical phenotypes. Images were taken using inverted phase contrast microscope after a week of plating. D Subspheres grown from LN229,

representative images of $3^{\text {rd }}$ and day $7^{\text {th }}$. Magnification $\times 40$. Scale bars represent $100 \mu \mathrm{m}$. E Single cell suspension of tumorsphere generated from LN229 cells or the parental cells were plated on coverslips and stained for SSEA1 antibody (green). Images were captured using confocal microscope. Cells were counterstained with Hoechst-33258 (blue.) Magnification $\times 40$, scale bars represent $50 \mu \mathrm{m}$. Experiment performed in duplicates and repeated thrice. Representative images are shown. $\boldsymbol{F}$ Glioblastoma spheres derived from a single mother cell were stained by NSC markers SOX2 (green) and OCT4 (Red). The images were taken using confocal microscope at $40 X$ magnification (with $2 x$ zoom). The Scale bar represents $50 \mu \mathrm{m}$. Nuclei were staining with Hoechst-33258 (blue.) Data represent results from triplicate experiments. 
Higher autophagy in tumorspheres as comparison with parental lines To determine the role of autophagy in GSC, firstly we evaluated the difference in the levels of autophagy between the parental/monolayer glioblastoma cells with their respective tumorspheres/GSC. To study the levels of autophagy, we determined autolysosomal activity using AO. AO is a protonated fluorophore that accumulates inside autolysosomes that are AVOs and gives red fluorescence due to metachromatic shift from green to red. An increase in red fluorescence is indicative of higher lysosomal activity associated with late stage of autophagy ${ }^{[16]}$. Using spectrofluorimetric analysis, we identified a significantly higher AO staining in tumorspheres as compared with the parental cells (Figure 2A). Further, since higher AO staining can be a result of defective removal of autolysosomes, we also analyzed autophagic flux using CQ a late stage autophagy inhibitor. AO staining was further enhanced in the presence of CQ in the GSC as compared with parental cells, indicative of higher autophagic flux (Figure 2A). These tumorspheres also maintained higher ROS levels as compared with monolayers as determined by DCFDA staining (Figure 2B). However, there was no significant difference in the MMP (Figure 2C) between the tumorspheres and their parental cultures (monolayer) which might be a positive indicator of cellular activation and proliferation in tumorspheres.
Next, we performed western blotting to analyze various autophagic proteins including LC-3, p62, ATG5, ATG7, ATG6/Beclin-1, ATG3 and ATG12 to determine the level of autophagy in GSCs (tumorspheres) as compared with the parental cells using whole cell protein lysates. LC-3 is the most reliable biomarker to study autophagic process where the cytoplasmic form LC3-I is converted to the lipidated form LC3-II which associates with the autophagosomes. This is an important step for the formation of autophagosomes and indicative of higher levels of autophagy. As shown in Figure 2D, an increase in the ratio of LC3-II/LC3-I was detected in the tumorspheres as compared with the parental cultures, indicative of higher autophagy. Correspondingly, western blotting with other autophagic marker proteins essential for autophagosome formation such as ATG5, Beclin-1, ATG7 showed relatively higher expression in tumorspheres as compared to the parental cells (Figure 2D). However, no significant change in ATG3 or ATG12 levels was observed between the parental cells and tumorspheres (data not shown), which may not be involved in CSC spheroids under normal conditions but might play a role during drug induced stress. Overall, these findings demonstrate higher levels of autophagy in GSCs as compared with non-GSC.
A

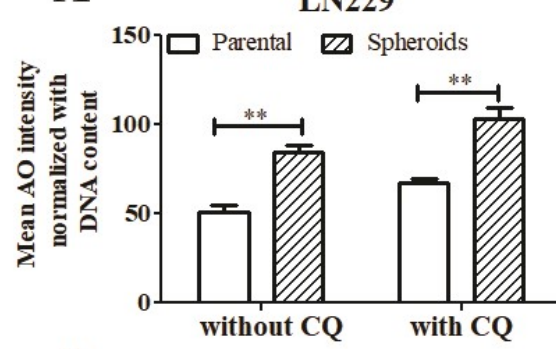

B
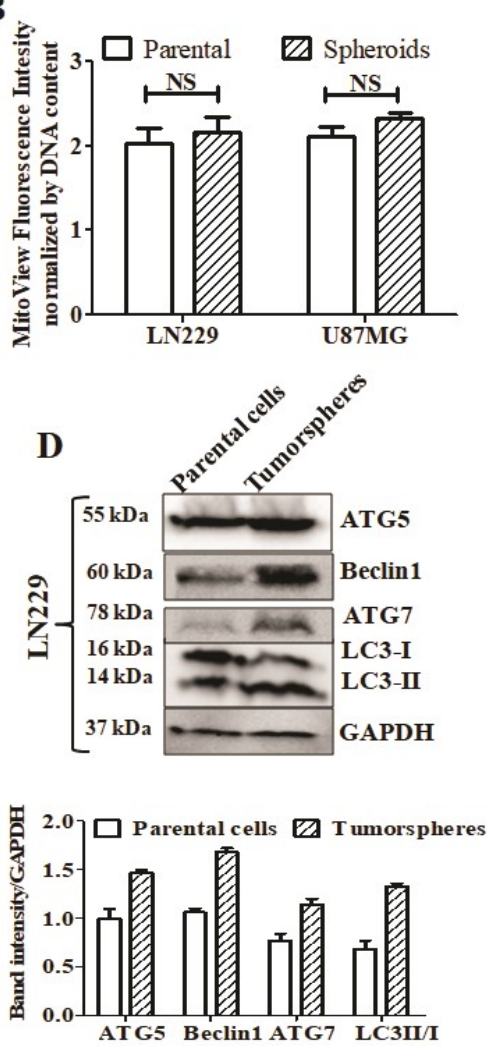

U87MG

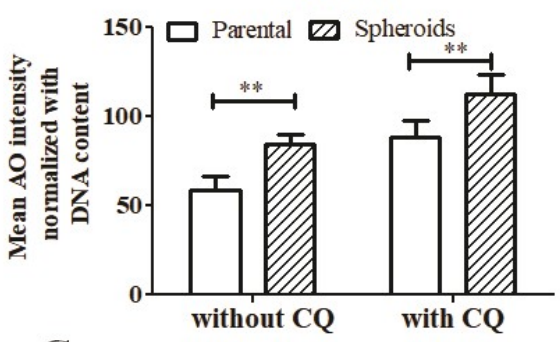

C
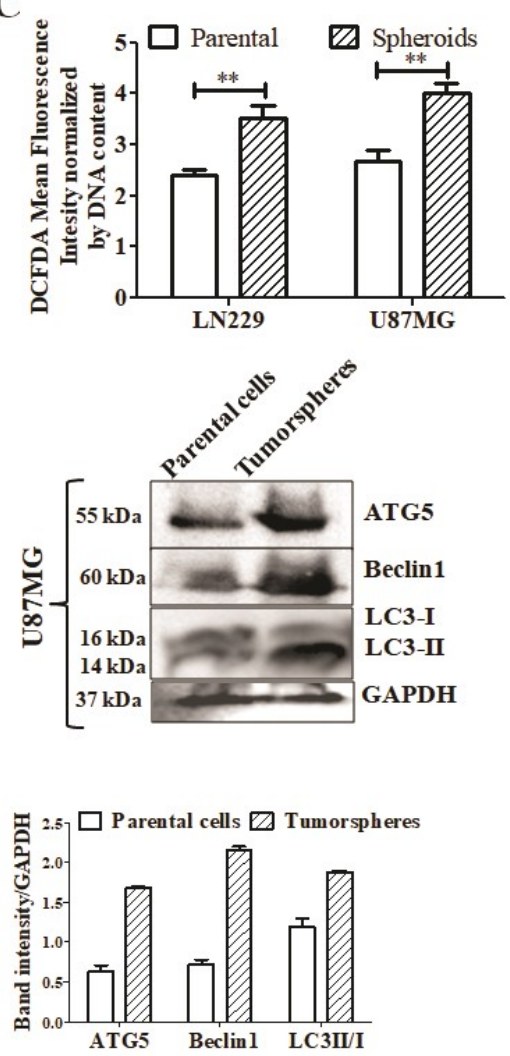

Figure. 2 The level of autophagy in glioblastoma tumorspheres (GSC) in comparison with adherent (parental) LN229 and U87MG cells. a Single cell suspension from tumorspheres and parental LN229 cells and U87MG cells that were treated with or without $C O$ to determine the flux for 2 hrs was prepared. A AO staining detected autophagic flux in the presence or absence of CQ in GSCs and adherent cells. Cells were stained with $A O$ for $30 \mathrm{~min}$, washed and analyzed by fluorescence plate reader. $A O$ staining corresponds to AVO formation. Single cell suspension from tumorspheres and parental LN229/U87MG cells were stained with $\boldsymbol{B}$ H2DCFDA for determining ROS generation or $C$ MitoViewTM for MMP for $30 \mathrm{~min}$, washed and analyzed by fluorescence plate reader. D Western blot analysis of the expression levels of LC3 I/II and ATG5, ATG7 and Beclin-1 in parental versus tumorspheres, GAPDH was used as a loading control. Densitometric analysis was performed using Image $J(\mathrm{NIH})$ and ratio of band intensity/GAPDH intensity are shown. Three independent experiments were performed and the results were expressed as the means \pm $S D$, and analyzed using one way ANOVA with Bonferroni posttests $(* P<0.05, * * P<0.01)$. 


\section{Modulation of autophagy affects the percentage of GSC population}

We further determined the role of higher levels of autophagy in regulating GSC population. Firstly, we treated the glioblastoma cell lines with autophagy inhibitor (3-MA and CQ, early and late stage inhibitors respectively) and determined the percentage of GSC in the total population. Side population is a subset of stem-like cells or progenitor cells that exhibit GSC-like properties and demonstrates week Hoechst-33342 dye staining pattern due to efflux of dye ${ }^{[17]}$. The SP's ability to exclude the dye was inhibited by using verapamil hydrochloride, an inhibitor of dye efflux and was used to standardize the SP assay which can discriminate them from the non-SP by creating appropriate gates during the analysis. The total percentage of GSC/progenitor cell population was reduced $\sim 2.5$ folds in the presence of autophagy inhibitors (Figure 3A). The control cells comprised of $1 \%-2 \%$ SP cells, this percentage was reduced ( $\sim 2.5$ folds) by autophagy inhibitors. Further, we treated the glioblastoma cells with autophagy inhibitors and western blotting was performed to determine the levels of cancer stem cell markers. Western blotting further confirmed that inhibition of autophagy was associated with a decrease in GSC pool (Figure 3B). Altogether, these results indicated that autophagy is required for the maintenance/existence of GSC pool in the parental glioblastoma cells.

Next, we investigated the function of autophagy in GSC population by studying its role in self-renewal and cell proliferation. Since the size and number of tumorspheres can be correlated with the selfrenewal and proliferation capacity, glioblastoma tumorspheres formation was studied following treatment with autophagy inhibitors 3-MA and CQ. Our results indicated that inhibition of autophagy significantly reduced the size as well as metabolic capacity/viability of the tumorspheres as compared to the controls (Figure 3C-D). Together, these results suggested that autophagy is critical for maintaining the GSCs population and its property of self-renewal and proliferation.
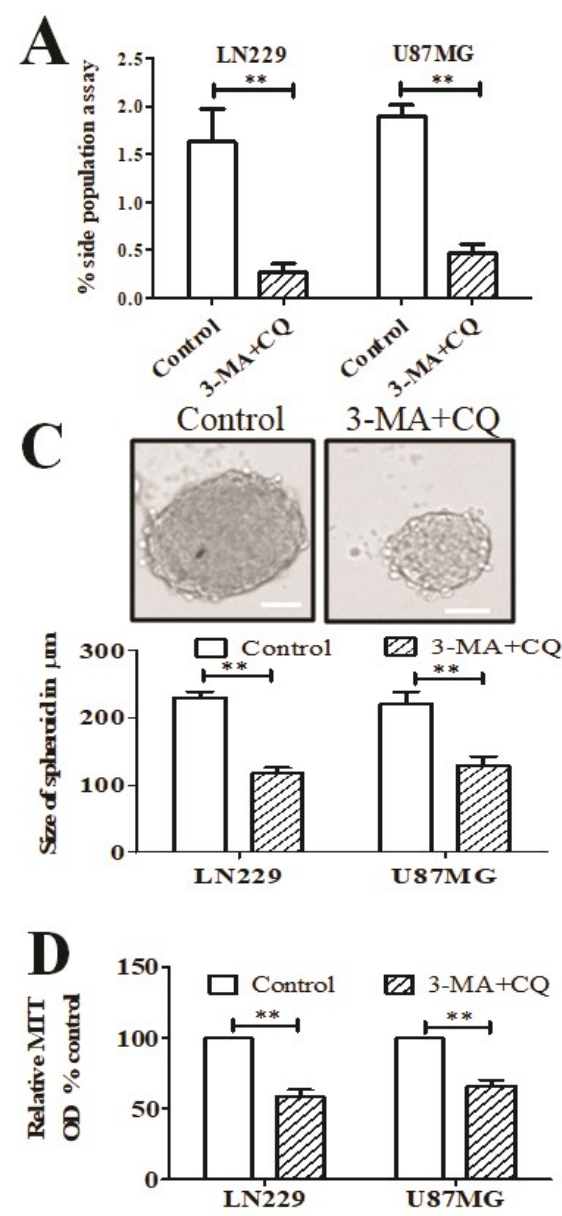

B

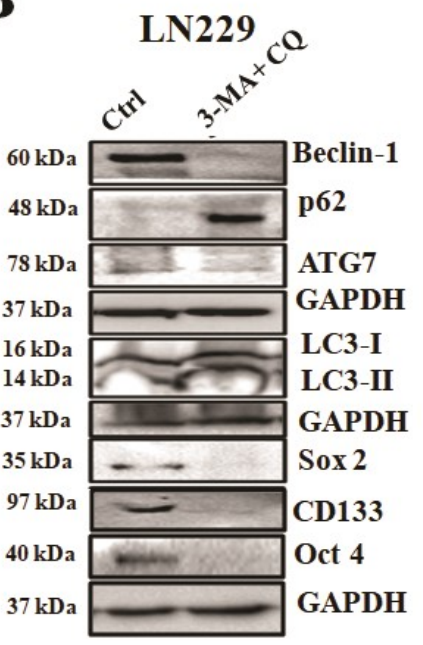

LN229

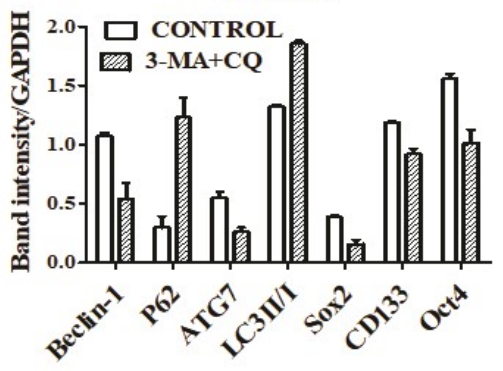

U87MG

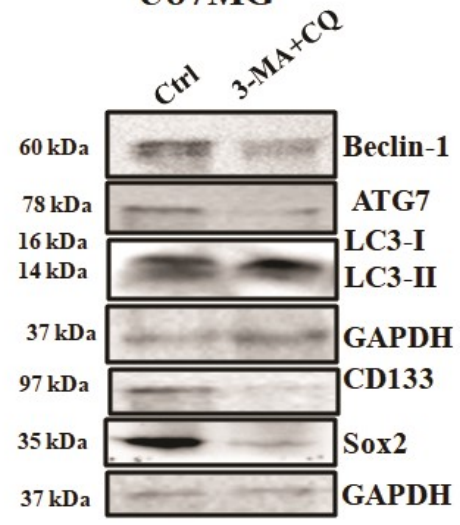

U87MG

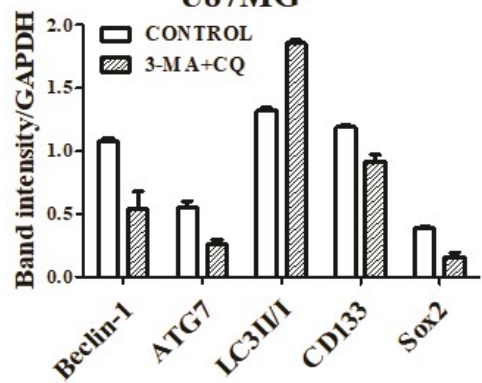

Figure. 3 Effects of autophagy on regulating the pool of cancer stem cells in parental cell population. A Flow-cytometry based SP assay in the presence or absence of autophagy inhibitor (3-MA+CQ) in the adherent LN229 and U87MG cells. Three independent experiments were performed and the results were expressed as the means $\pm S D$, and analyzed using Student's t-test $(* P<0.05, * * P<0.01)$. B Western blotting confirmed the autophagy inhibition by 3-MA $+C Q$ as shown by various autophagy markers (BECLIN1, p62, ATG7 and LC3-I/II ratio); expression levels of pluripotency-associated proteins (SOX2, CD133 and OCT4) are also shown in adherent LN229 and U87MG cells in the presence or absence of autophagy inhibitor (3-MA+CO) treatment for 72 hrs. GAPDH is used as loading control. Densitometric analysis was performed using Image $J$ (NIH) and ratio of band intensity/GAPDH intensity are shown. C-D Effect of autophagy inhibition on self renewal and proliferation capacity of GSC. C Representative images of LN229 tumorspheres along with size measurement (in $\mu \mathrm{m}$ ) for LN229 and U87MG cells. D. Proliferation/viability of GSCs by MTT assay, in the presence of absence of 3-MA and $C Q$ treatment after 7 days. Experiments were performed in triplicates and repeated at least twice obtaining similar results. Results were expressed as the means \pm SD, and analyzed using one way ANOVA with Bonferroni posttests $(* P<0.05, * * P<0.01)$. 


\section{TMZ induces survival autophagy}

It has been reported that glioblastoma cells preferentially activate autophagic process during therapeutic stress ${ }^{[10,14,18-21]}$. In addition, autophagy is induced by therapeutic drug TMZ at higher concentrations $\left(\mathrm{IC}_{50}\right)$ in glioblastoma cell lines, which lead to cell death. However, contribution of autophagy at clinical relevant and lower dosage of TMZ has not been studied earlier. Therefore, we investigated the potential role of lower dosage of TMZ on cell viability as well as autophagy induction in glioblastoma cells. Thus, LN229 and U87MG cells were treated with different concentrations of TMZ $(0-200 \mu \mathrm{M})$ for $48 \mathrm{hrs}$. Cell viability was analyzed by PI and Hoechst-33258 staining. We did not find significant cell death at TMZ concentrations ranging from $0-100 \mu \mathrm{M}$ and significant cell death was induced only at higher concentration $200 \mu \mathrm{M}$ (Figure 4A). Since we were interested in studying clinically achievable concentrations, we selected $50 \mu \mathrm{M} \mathrm{TMZ}$ (physiological levels of TMZ concentration in the plasma of the patients who underwent chemotherapy) to further study autophagy induction in glioblastoma cells. AO staining was performed to study the lysosomal activity an indicator of autophagy and western blotting was performed to study the bonafide markers of autophagy that included LC3-I to LC3-II conversion and levels of P62, ATG3, ATG6/Beclin-1, ATG7 after treatment with $\mathrm{TMZ}$ in the presence or absence of autophagy inhibitors (Figure 4B-C). Treatment of glioblastoma cells with TMZ resulted in up-regulation of $\mathrm{AO}$ staining (Figure 4B). Western blotting results also indicated higher conversion of LC3-II along with an increase in LC3-I levels (as can be seen as higher autophagy flux in TMZ+CQ treated cells), loss of p62, upregulation of ATG3, ATG6 and ATG7, suggesting up-regulation of autophagy upon TMZ treatment even at lower concentration in LN229 cells (Figure 4C). Similarly, upregulation of autophagy was observed in U87MG cells as determined LC3II/I ration and upregulation of various autophagic marker proteins as determined by western blotting (Figure 4C). These results indicated that autophagy is activated in glioblastoma cells at lower TMZ concentrations, at which no significant cell death was observed. We hypothesized that at such lower dosage of TMZ autophagy might be activated as a survival response and inhibition of autophagy may increase the efficacy of the TMZ. In line with our hypothesis, combination of TMZ with autophagy inhibitors (CQ and 3-MA) significantly augmented the toxicity of TMZ in both the cell lines as determined by cell viability assay using PI (Figure 4D). These results suggest that autophagy is induced in TMZ as a survival response and inhibition of autophagy can increase the susceptibility of glioblastoma cells towards TMZ even at sub-toxic dosage.
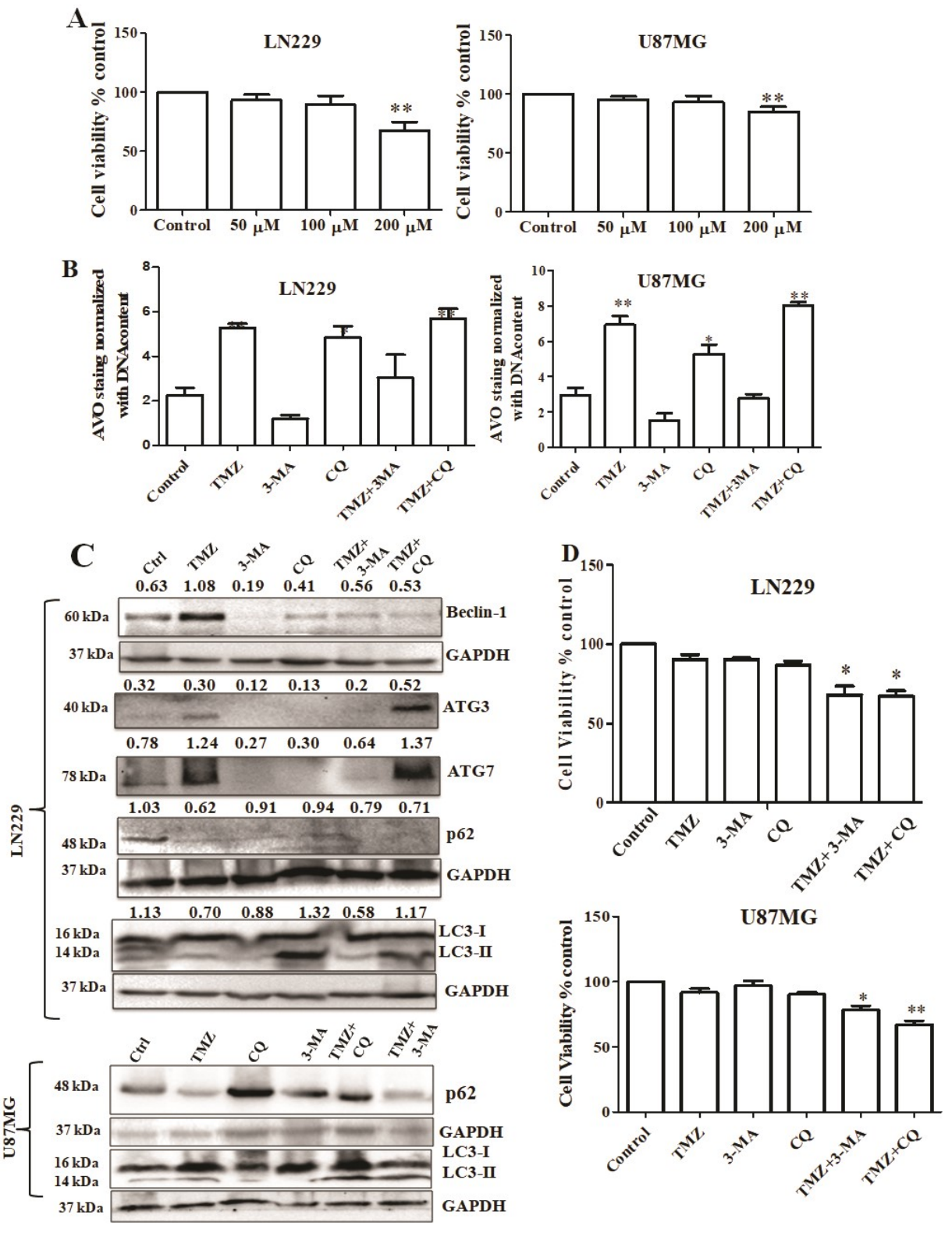

Figure. 4 Lower dosage of TMZ can induce survival autophagy. A Cell viability (PI staining) to study cytotoxicity of TMZ on LN229 and U87MG cells. Cells were treated with different dosage of $T M Z(0-200 \mu M)$ for 72 hrs followed by PI and Hoechst-33258 staining and spectrofluorimetric analysis was performed. Experiment performed in triplicates and data presented as cell viability percent control. Experiment repeated at least three times. B AO staining to detect autophagic flux in $T M Z(50 \mu M)$ treated in the presence of absence of 3 $M A(1 \mathrm{mM})$ or CQ $(20 \mu M)$ in LN229 and U87MG cells for 24 hrs. Cells were stained with AO for 30 min, washed and analyzed by fluorescence plate reader. AO staining corresponds to AVO formation and data presented relative to DNA content (green fluorescence of $A O)$. C Western blotting to detect the level of autophagy marker proteins in LN229 and U87MG cells treated with TMZ $(50 \mu M)$ in the presence of absence of 3-MA (1 mM) or CQ $(20 \mu \mathrm{M})$ for 72 hrs. GAPDH was used as loading control. Representative images are presented. Band intensities were determined using Image $J(\mathrm{NIH})$ analysis and the values normalized by respective $G A P D H$ intensities are presented along with the blots. D Cell viability determined by PI staining \% control after treatment with TMZ $(50 \mu M)$ in the presence of absence of 3-MA and CO in LN229 and U87MG cells after 72 hrs. Three independent experiments were performed and the results were expressed as the means $\pm S D$, and analyzed using one way ANOVA with Bonferroni posttests $(* P<0.05, * * P<0.01$ in comparison with control) 
Autophagy plays a role in TMZ-induced GSC pool in glioblastoma cells

TMZ has been shown to induce conversion of non-GSC to GSC at clinically achievable concentrations which may account for tumor relapse ${ }^{[11,21]}$. Since our findings indicated a role of autophagy in GSC self renewal and maintenance, we further studied the role of autophagy in altering the pool of GSC in response to TMZ treatment. Glioblastoma cells LN229 and U87MG were treated with TMZ alone or combination with CQ or 3-MA for 3 days and GCS markers were studied by western blotting (Figure 5A). Our results indicated that TMZ treatment up-regulated the level of GSCs markers that correlated with an increase in autophagy (Figure 5A and Figure 4B) and inhibition of autophagy inhibited the increases in GSC markers in TMZ treated cells (Figure 5A).We further determined the effect of TMZ on spheroid forming capacity that can directly correlate the effect of TMZ on GSCs pool. TMZ inhibited the tumorsphere forming capacity as determined by reduction in the size and metabolic activity of the spheroids which was further augmented by combination of autophagy inhibitors (Figure 5B-C). Thus, inhibition of autophagy not only increases the cytotoxic effect of TMZ but also significantly inhibits the increase in GSC pool in response to $\mathrm{TMZ}$ treatment.

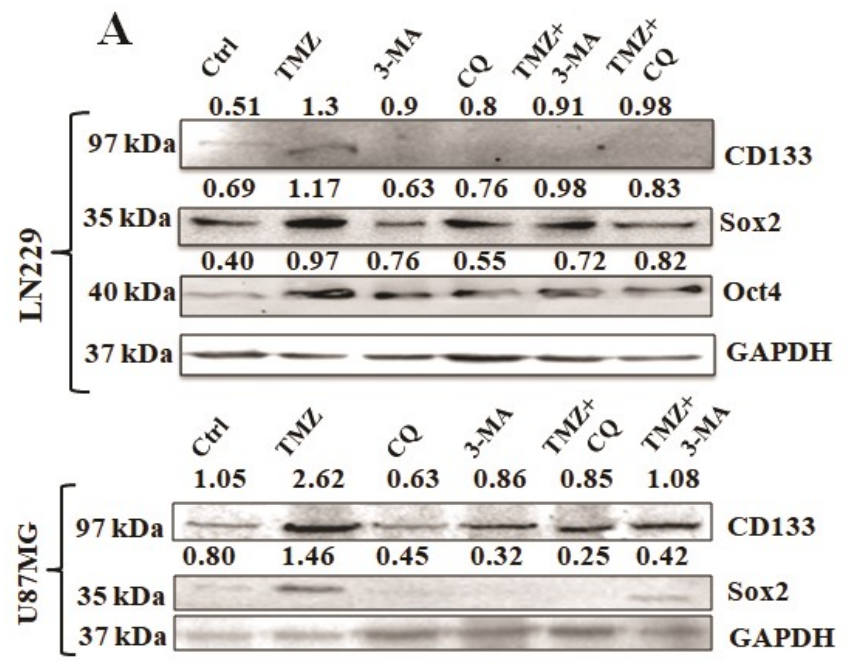

$\mathbf{B}$

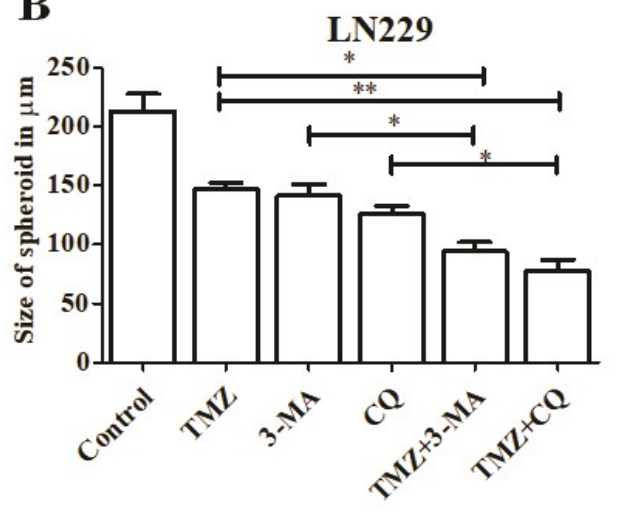

C

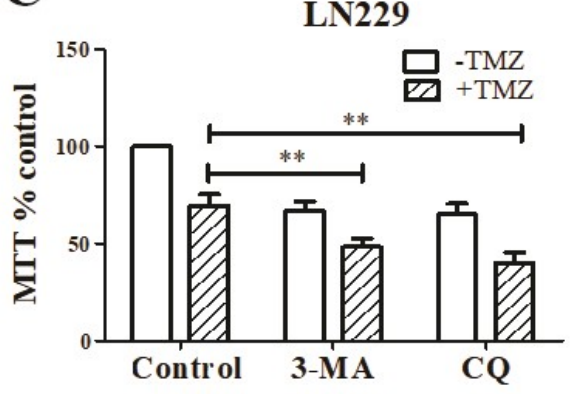

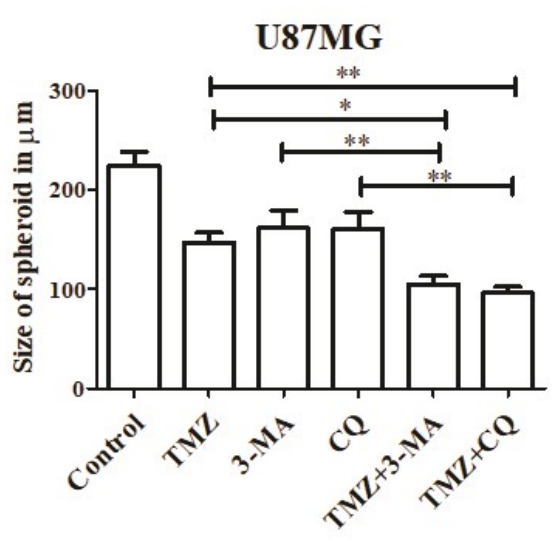

U87MG

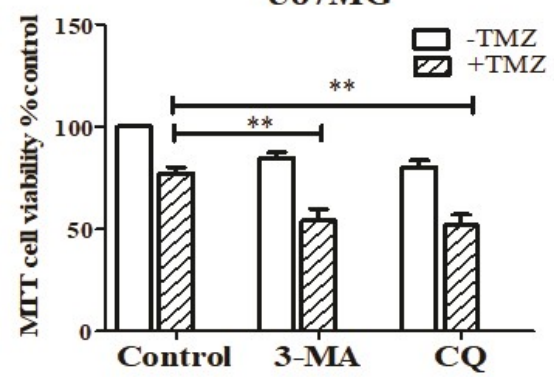

Figure. 5 Role of autophagy in TMZinduced upregulation of pool of GSC: A. $L N 229$ and U87MG adherent cultures were treated with $50 \mu M$ of TMZ in the presence or absence of 3-MA (1 $\mathrm{mM})$ or $C Q(20 \mu M)$ for 72 hrs. Western blotting was performed to analyze the GSC pool in the cellular lysate PVDF membranes were probed for CSC markers. GAPDH was used a loading control. Representative images are presented. Band intensities were determined using Image $J(N I H)$ analysis and the values normalized by respective GAPDH intensities are presented along with the blots. Experiment was repeated at least twice. B-C LN229 and U87MG cells were grown as tumorspheres in low attachment plates at a density of 1500 cells/well in 96 well plate. After 24 hrs of plating cells were treated with $50 \mu \mathrm{M}$ of $T M Z$ in the presence or absence of 3-MA $(1 \mathrm{mM})$ or $C Q(20 \mu \mathrm{M})$ and allowed to grow for 7 days with change in media on the $3^{\text {rd }}$ day. B Size of tumorsphere were calculated (150 $4 m-250 \quad \mu m)$ and presented in histogram. C Cellular proliferation/viability was measured by MTT assay in spheroids. Experiment was performed in triplicates and repeated at least twice. Three independent experiments were performed and the results were expressed as the means $\pm S D,(* P<0.05, * * P<0.01)$ 


\section{Discussion}

Emerging concept suggest GSCs are responsible for initiation, tumor heterogeneity, progression, therapy resistance and recurrence of HGG. Thus, it becomes essential to identify the molecular mechanism that lead to the emergence and maintenance of GSCs to develop newer effective therapeutic approaches that can target GSC population. In this study, we provide evidence that autophagy is higher in GSCs and regulate its functions. Autophagy is induced at suboptimal, clinically achievable dosage of TMZ. Modulation of autophagy can enhance the therapeutic effect of TMZ by inhibiting the pool of GSCs that are generated in response to TMZ treatment, one of the current standards of care treatments in anti-glioma therapy.

To study the role of autophagy in GSCs, we generated tumorspheres from glioblastoma cell lines that are well established GSC model. Due to intra-tumoral variation and tissue heterogeneity multiple GSC markers were employed for identification of GSC that included surface markers such as CD133, CD15 combined with other stem cell markers such as SOX2, and NANOG as suggested in literature ${ }^{[22]}$.Using these markers, we were able to identify GSCs as well as monitor changes in the population of these GSC in response to autophagy inhibition. Indeed, tumorspheres showed increased expression of several autophagy markers as well as AVO formation as compared to non-GSCs. Further, we observed that although GSCs in tumorspheres maintain higher level of ROS, it maintained MMP comparable to their parental cultures which indicate a healthy proliferative state in GSCs. Additionally, autophagy induction in GSCs might be involved in removal of unhealthy mitochondria/ proteins and stressors, thereby preventing the loss of MMP and maintain cells in a healthy state despite of elevated ROS levels. Interestingly, upregulation of autophagy promoted GSC population and inhibition of autophagy significantly depleted the pool of GSC population, suggesting dependence of GSC population on autophagy. In line with these observations the size and number of tumorsphere was also decreased when autophagy was blocked by 3 MA and CQ at an early and late stage respectively. Previous studies also suggested that like tissue stem cells, CSCs also show autophagy dependence ${ }^{[7]}$. Reliance of CSC on autophagy has been most studied in breast cancer CSCs and also been implicated in other cancer types, including pancreatic cancer, bladder cancer, colorectal cancer, chronic myeloid leukemia and glioblastoma, ovarian carcinoma ${ }^{[23-29]}$. These findings indicate towards the role of autophagy that may help to maintain the CSC pool, which may be crucial for developing cancer relapse. However, the pathways that induce autophagy in CSCs and its role in stemness remain to be explored further.

Besides role in various processes, cancer therapy is known to induce autophagy that involves ROS-induced Forkhead box O (FOXO) and activating transcription factor-4 (ATF4) transcription which activate autophagy genes like ATG5, LC3 and others involved in autophagic pathways $^{[30]}$. In majority of cases autophagy induction promotes cell survival and provides resistance to therapy. Of interest, it has been identified that glioma cells are resistance to therapy-induced apoptosis and preferably activate autophagic process. Autophagy induction has been implicated in the response to TMZ, the most commonly used anti-glioma chemotherapeutic agent, radiotherapy as well as to molecularly targeted therapies in $\mathrm{HGG}^{[10,14,18-21]}$. Presently, our results also demonstrated that autophagy was induced at clinically relevant dosage of TMZ and promoted survival of glioblastoma cells. Interestingly, it has been observed that exposure of glioblastoma cell lines to therapeutic doses of TMZ, there is an increase in population of GSCs over time both in-vitro and in-vivo, that may account for relapse ${ }^{[11]}$. In the present study, our experimental data also provided evidence of an increase in the population of GSCs in response to TMZ treatment at clinically relevant dosage. Since TMZ has been identified to induce autophagy in glioblastoma cells, we hypothesized that induction of autophagy in response to TMZ might be responsible to drive the pool of GSC population in response to TMZ treatment and inhibition of autophagy may suppress the pool of induced GSCs. In line with our hypothesis, we observed that inhibition of autophagy indeed inhibited the increase in the pool of GSC population in response to TMZ treatment. Thus, present study, reports role of autophagy in regulating the pool of GSCs in response to therapy where inhibition of autophagy can inhibit TMZ-induced GSC pool. Interestingly, Auffinger et al, in their study also demonstrated conversion of nonGSCs into GSCs in response to TMZ treatment by increasing plasticity of non-GSC ${ }^{11]}$. Their study proposed mechanisms of cellular plasticity behind the increase and maintenance of GSCs post chemotherapy. However, it is not clear how non-GSCs are stimulated to dedifferentiate and increase the overall 'stemness' of the tumor. Our present study provides a possible explanation through involvement of autophagy in therapy-induced generation of stem cell pool. To our knowledge, this is the first time that the role of autophagy has been identified in regulating GSC pool in response to TMZ treatment. Such a finding provides very important information that adds up to the current understanding of the origin, development, and maintenance of the GSC population post-TMZ treatment.

Interestingly, our results demonstrated that tumorspheres were sensitive to TMZ-induced cell death which was further enhanced by combining autophagy inhibitors. These results indicate that the increase in TMZ-induced GSC population may not be due to expansion of chemo-resistant GSC population but due to de differentiation of its non-GSC counterpart. These findings are also in line with previous report by Beier et al., which suggested that GSC are sensitive to $\mathrm{TMZ}$ treatment ${ }^{[31]}$ and supports the previous theory of plasticity that suggested that CSC pool was generated from nonGSC part due to plasticity as proposed by Auffinger et al. ${ }^{[11]}$.

Thus, present study strongly supports rationale to combine cancer therapeutic approaches with agents that inhibit autophagy ${ }^{[7,32]}$. Late stage autophagy inhibitor CQ or hydroxychloroquine (HCQ) is a FDA-approved drug used in clinical setting, accumulates in lysosomes and act by inhibition of lysosomal acid protease activity ${ }^{[32,33]}$. Of interest, in clinical settings, combining TMZ with CQ has doubled patient survival times as compared to TMZ alone ${ }^{[34,35]}$. However, due to side effects, designing more potent and specific analogs is required for improving efficacy and to target specific cell type such as GSCs. Future studies are targeted to validate the TMZ-induced increase in GSC pool, in TMZ-treated patient derived glioblastoma cells. Our finding indicate that these GSCs pool may be generated due to conversion of non-GSCc to GSC via autophagy up-regulation, and anticipate that in-vivo investigations in patient- derived cells will indicate direct functional role of autophagy mechanism in generation of GSCs during TMZinduced therapeutic response.

Furthermore, with growing amount of evidences that propose CSCs can be induced from non-CSCs by radio/chemotherapy and lead to tumor recurrence and metastasis ${ }^{[36]}$; it becomes crucial to investigate if autophagy contributes in such conversion in other cancer types and if autophagy inhibition can prevent dedifferentiation of non-CSCs to CSCs when combined with known radio/chemotherapeutic regime to improve antineoplastic efficacy in different tumor types.

\section{Conclusion}

Overall, the present study identifies that autophagy plays a significant role in GSC population. Autophagy modulation when combined with TMZ can: 1) increase the cytotoxic effect of TMZ; and 2) it can significantly inhibit the increase in the GSCs pool in response to TMZ treatment. Thus, autophagy modulation can improve the outcome of the TMZ treatment regime not only by increasing the efficacy but also may be useful in preventing residual disease and recurrence. Overall, our present study contributes to defining a critical role of autophagy in GSCs that maybe implicated in the design and interpretation of clinical trials. 


\section{References}

1. Mirimanoff RO. High-grade gliomas: reality and hopes. Chin J Cancer. 2014;33(1):1-3.

2. Stupp R, Mason WP, van den Bent MJ, et al. Radiotherapy plus concomitant and adjuvant temozolomide for glioblastoma. N Engl J Med. 2005;352(10):987-96.

3. Tentori L, Graziani G. Recent approaches to improve the antitumor efficacy of temozolomide. Curr Med Chem. 2009;16(2):245-57.

4. Kitange GJ, Carlson BL, Schroeder MA, Grogan PT, Lamont JD, Decker PA, Wu W, James CD, Sarkaria JN. Induction of MGMT expression is associated with temozolomide resistance in glioblastoma xenografts. Neuro Oncol. 2009;11(3):281-91.

5. Dwivedi AR, Thakur A, Kumar V, et al. Targeting Cancer Stem Cells Pathways for the Effective Treatment of Cancer. Curr Drug Targets. 2020;21(3):258-278.

6. Nunes T, Hamdan D, Leboeuf C, El Bouchtaoui M, Gapihan G, Nguyen TT, Meles S, Angeli E, Ratajczak P, Lu H, Di Benedetto M, Bousquet G, Janin A. Targeting Cancer Stem Cells to Overcome Chemoresistance. Int J Mol Sci. 2018 ;19(12):4036.

7. Smith AG, Macleod KF. Autophagy, cancer stem cells and drug resistance. J Pathol. 2019;247(5):708-718.

8. Buccarelli M, Marconi M, Pacioni S, De Pascalis I, D'Alessandris QG, Martini M, Ascione B, Malorni W, Larocca LM, Pallini R, Ricci-Vitiani L, Matarrese P. Inhibition of autophagy increases susceptibility of glioblastoma stem cells to temozolomide by igniting ferroptosis. Cell Death Dis. 2018;9(8):841.

9. Rosenfeld MR, Ye X, Supko JG, Desideri S, Grossman SA, Brem S, Mikkelson T, Wang D, Chang YC, Hu J, McAfee Q, Fisher J, Troxel AB, Piao S, Heitjan DF, Tan KS, Pontiggia L, O'Dwyer PJ, Davis LE, Amaravadi RK. A phase I/II trial of hydroxychloroquine in conjunction with radiation therapy and concurrent and adjuvant temozolomide in patients with newly diagnosed glioblastoma multiforme. Autophagy. 2014;10(8):1359-68.

10. Yan Y, Xu Z, Dai S, Qian L, Sun L, Gong Z. Targeting autophagy to sensitive glioma to temozolomide treatment. J Exp Clin Cancer Res. 2016;35:23.

11. Auffinger B, Tobias AL, Han Y, Lee G, Guo D, Dey M, Lesniak MS, Ahmed AU. Conversion of differentiated cancer cells into cancer stem-like cells in a glioblastoma model after primary chemotherapy. Cell Death Differ. 2014;21(7):1119-31.

12. Gao W, Wu D, Wang Y, Wang Z, Zou C, Dai Y, Ng CF, Teoh JY, Chan FL. Development of a novel and economical agar-based non-adherent three-dimensional culture method for enrichment of cancer stem-like cells. Stem Cell Res Ther. 2018;9(1):243

13. Ruan Z, Liu J, Kuang Y. Isolation and characterization of side population cells from the human ovarian cancer cell line SK-OV3. Exp Ther Med. 2015;10(6):2071-2078.

14. Tiwari M, Bajpai VK, Sahasrabuddhe AA, Kumar A, Sinha RA, Behari S, Godbole MM. Inhibition of N-(4hydroxyphenyl)retinamide-induced autophagy at a lower dose enhances cell death in malignant glioma cells. Carcinogenesis. 2008;29(3):600-9.

15. Ishiguro T, Ohata H, Sato A, Yamawaki K, Enomoto T, Okamoto K. Tumor-derived spheroids: Relevance to cancer stem cells and clinical applications. Cancer Sci. 2017;108(3):283-289.

16. Thomé MP, Filippi-Chiela EC, Villodre ES, Migliavaca CB, Onzi GR, Felipe KB, Lenz G. Ratiometric analysis of Acridine Orange staining in the study of acidic organelles and autophagy. J Cell Sci. 2016;129(24):4622-4632.

17. Challen GA, Little MH. A side order of stem cells: the SP phenotype. Stem Cells. 2006;24(1):3-12.

18. Geng Y, Kohli L, Klocke BJ, Roth KA. Chloroquine-induced autophagic vacuole accumulation and cell death in glioma cells is p53 independent. Neuro Oncol. 2010;12(5):473-81.
19. Kanzawa T, Germano IM, Komata T, Ito H, Kondo Y, Kondo S. Role of autophagy in temozolomide-induced cytotoxicity for malignant glioma cells. Cell Death Differ. 2004;11(4):448-57.

20. Kanzawa T, Zhang L, Xiao L, Germano IM, Kondo Y, Kondo S. Arsenic trioxide induces autophagic cell death in malignant glioma cells by upregulation of mitochondrial cell death protein BNIP3. Oncogene. 2005;24(6):980-91.

21. Lee SW, Kim HK, Lee NH, Yi HY, Kim HS, Hong SH, Hong YK, Joe YA. The synergistic effect of combination temozolomide and chloroquine treatment is dependent on autophagy formation and p53 status in glioma cells. Cancer Lett. 2015;360(2):195-204.

22. $\mathrm{Xu} \mathrm{HS}$, Qin XL, Zong HL, He XG, Cao L. Cancer stem cell markers in glioblastoma - an update. Eur Rev Med Pharmacol Sci. 2017;21(14):3207-3211.

23. Galavotti S, Bartesaghi S, Faccenda D, Shaked-Rabi M, Sanzone S, McEvoy A, Dinsdale D, Condorelli F, Brandner S, Campanella M, Grose R, Jones C, Salomoni P. The autophagy-associated factors DRAM1 and p62 regulate cell migration and invasion in glioblastoma stem cells. Oncogene. 2013;32(6):699-712.

24. Gong C, Bauvy C, Tonelli G, Yue W, Deloménie C, Nicolas V, Zhu Y, Domergue V, Marin-Esteban V, Tharinger H, Delbos L, Gary-Gouy H, Morel AP, Ghavami S, Song E, Codogno P, Mehrpour M. Beclin 1 and autophagy are required for the tumorigenicity of breast cancer stem-like/progenitor cells. Oncogene. 2013;32(18):2261-72, 2272e.1-11.

25. Maycotte P, Jones KL, Goodall ML, Thorburn J, Thorburn A. Autophagy Supports Breast Cancer Stem Cell Maintenance by Regulating IL6 Secretion. Mol Cancer Res. 2015;13(4):651-8.

26. Peng Q, Qin J, Zhang Y, Cheng X, Wang X, Lu W, Xie X, Zhang S. Autophagy maintains the stemness of ovarian cancer stem cells by FOXA2. J Exp Clin Cancer Res. 2017;36(1):171.

27. Rausch V, Liu L, Apel A, Rettig T, Gladkich J, Labsch S, Kallifatidis G, Kaczorowski A, Groth A, Gross W, Gebhard MM, Schemmer P, Werner J, Salnikov AV, Zentgraf H, Büchler MW, Herr I. Autophagy mediates survival of pancreatic tumourinitiating cells in a hypoxic microenvironment. $J$ Pathol. 2012;227(3):325-35.

28. Sharif T, Martell E, Dai C, Kennedy BE, Murphy P, Clements DR, Kim Y, Lee PW, Gujar SA. Autophagic homeostasis is required for the pluripotency of cancer stem cells. Autophagy. 2017;13(2):264-284.

29. Yeo SK, Guan JL. Hierarchical heterogeneity in mammary tumors and its regulation by autophagy. Autophagy. 2016;12(10):19601961.

30. Füllgrabe J, Ghislat G, Cho DH, Rubinsztein DC. Transcriptional regulation of mammalian autophagy at a glance. J Cell Sci. 2016;129(16):3059-66.

31. Beier D, Röhrl S, Pillai DR, Schwarz S, Kunz-Schughart LA, Leukel P, Proescholdt M, Brawanski A, Bogdahn U, TrampeKieslich A, Giebel B, Wischhusen J, Reifenberger G, Hau P, Beier CP. Temozolomide preferentially depletes cancer stem cells in glioblastoma. Cancer Res. 2008;68(14):5706-15.

32. Levy JMM, Towers CG, Thorburn A. Targeting autophagy in cancer. Nat Rev Cancer. 2017;17(9):528-542.

33. Pascolo S. Time to use a dose of Chloroquine as an adjuvant to anti-cancer chemotherapies. Eur J Pharmacol. 2016;771:139-44.

34. Briceno E, Reyes S, Sotelo J. Therapy of glioblastoma multiforme improved by the antimutagenic chloroquine. Neurosurg Focus. 2003;14(2):e3.

35. Sotelo J, Briceno E, Lopez-Gonzalez MA. Adding chloroquine to conventional treatment for glioblastoma multiforme: a randomized, double-blind, placebo-controlled trial. Ann Intern Med. 2006; 144(5):337-43.

36. Chen X, Liao R, Li D, Sun J. Induced cancer stem cells generated by radiochemotherapy and their therapeutic implications. Oncotarget. 2017;8(10):17301-12. 


\section{Abbreviations}

$\begin{array}{llll} & & \text { MGMT } & \text { : O6 methylguanine-DNA methyl transferase enzyme } \\ \text { 3-D } & : \text { 3-Dimensional } & \text { MMP } & : \text { Mitochondrial Membrane Potential } \\ \text { 3-MA } & : \text { 3-Methyl adenine } & \text { MTT } & \text { tetraz-dimethylthiazol-2-yl]-2,5 diphenyl } \\ \text { AO } & : \text { Acridine Orange } & & \text { : Propidium iodide } \\ \text { AVO } & : \text { Acidic Vacuolar Organelles } & \text { PI Polyvinilidenedifluoride } \\ \text { CQ } & : \text { Chloroquine } & \text { PVDF } & : \text { Reactive Oxygen Species } \\ \text { CSCs } & : \text { Cancer Stem Cells } & \text { ROS } & : \text { Room Temperature } \\ \text { DMEM } & : \text { Dulbecco's Modified Eagle Medium } & \text { RT } & : \text { Standard Deviation } \\ \text { GSCs } & : \text { Glioma stem cells } & \text { SD } & : \text { Sodium Dodecyl Sulfate } \\ \text { H2DCFDA } & : 2^{\prime}, 7^{\prime} \text {-Dichlorodihydrofluorescein diacetate } & \text { SDS } & : \text { Side Population } \\ \text { HGG } & : \text { High Grade Glioma } & \text { SP } & \text { Temozolomide }\end{array}$

\section{Potential Conflicts of Interests}

None

\section{Acknowledgement:}

The authors would like to acknowledge the funding support from Department of Biotechnology Bio-CARe grant, Govt. of India (BT/P19357/BIC/101/927/2016 to M.T.) and Indian Council of Medical Research, Govt. of India (2019-0665/CMB/Adhoc/BMS to L.K.S.).

\section{Ethical standards}

All experiments were conducted in compliance with the ethics committee of AIIMS-Patna and SGPGIMS-Lucknow.

\section{Additional Information}

Supplementary Information accompanies this article. Supplementary information is linked to the online version of the article.

\section{Corresponding Authors}

1. Meenakshi Tiwari, Department of Pathology/ Lab Medicine, All India Institute of Medical Sciences-Patna, Phulwarisharif, Patna, Bihar801507, India; Email: meenakshimani79@yahoo.co.in,meenakshimani79@gmail.com

2. Lokendra Kumar Sharma, Department of Molecular Medicine \& Biotechnology, Sanjay Gandhi Post Graduate Institute of Medical Sciences, Rai Barely Road Lucknow, (U.P.)-226014, India; Email: rajlokendra@yahoo.co.in, lokendra@sgpgi.ac.in 